\title{
Retracted: \\ Long Noncoding RNA SOX2OT Accelerates the Carcinogenesis of Wilms' Tumor Through ceRNA Through miR-363/FOXP4 Axis
}

\author{
Li Ma, Xingzhen Sun, Wenxia Kuai, Jian Hu, Yufang Yuan, Weijing Feng, and Xincui Lu
}

It has come to the Journal's attention that the cell lines used in the study titled "Long Noncoding RNA SOX2OT Accelerates the Carcinogenesis of Wilms' Tumor Through ceRNA Through miR-363/FOXP4 Axis" by Ma L, Xingzhen S, Kuai W, Hu J, Yuan Y, Feng W, and Lu X (DNA Cell Biol [Epub ahead of print]; DOI: 10.1089.dna.2018.4420), are not of Wilms' tumor origin, but rather are Ewing (SK-NEP-1) (Memorial Sloan Kettering) and Rhabdoid (G-401) (ATCC). These data were provided by unimpeachable authorities. Memorial Sloan Kettering Cancer Center (among the premier institutions for cancer research in the world) and ATCC (the most highly respected repository for cell lines and reagents) disagree with the authors assumptions about the specificity of the origin of the cell lines.

While many other publications can be found that assert the cell lines are of Wilms' tumor origin, this is not supported by the benchmark organizations.

For that reason, the experimental conclusions drawn in this peer-reviewed paper cannot be attributed to provide insights into Wilms' tumors, but rather to two cell lines of other pedigree. Therefore, DNA and Cell Biology is officially retracting this paper.

\section{References}

ATCC. www.lgcstandards-atcc.org/Products/All/CRL-1441.aspx?geo_country=it

Memorial Sloan Kettering Cancer Cancer. https://www.mskcc.org/research-advantage/support/technology/tangi blematerial/sk-nep-1-human-ewing-sarcoma-cell-line

Keywords: Wilms' tumor, SOX2OT, miR-363, FOXP4

Introduction

W ILMS' TUMOR is one of the most common malignant tumors in children, which is also known as embryonal tumor or renal embryonal tumor (Gonzalez-Urquijo et al., 2018; Guerreiro et al., 2018). In fact, Wilms' tumor is the high widespread solid tumors (Petiti et al., 2018). The incidence of Wilms' tumor causes serious influence on children's health (Totadri et al., 2017). Every year, Wilms tumor triggers thousands of fatality rate. Therefore, it is urgently necessary to investigate the pathogenesis and tumorigenesis of Wilms' tumor (Wang et al., 2018a).

Long noncoding RNAs (IncRNAs) are a type of transcription products $>200 \mathrm{nt}$ length in human genome (Lv et al., 2018; Sun et al., 2018a). The roles of lncRNAs in human diseases have been renewedly recognized in decades, especially in human cancers (Zhang et al., 2017a). lncRNAs are implicated in series of molecular processes, including differentiation, proliferation, metastasis, and transcriptional regulation. For instance, IncRNA LINC00460 is significantly upregulated in meningioma tissues and cells, and it promotes MMP-9 expression through targeting miR-539, thereby accelerating the pathogenesis (Xing et al., 2018). IncRNA UCA1 antagonizes miR-26a and recovers its target PETN to alleviate VSMCs proliferation against atherosclerosis (Tian et al., 2018).

In several human cancers, IncRNA SOX2OT has been reported to function as oncogenic molecular, such as esophageal squamous cell carcinoma (Shafiee et al., 2016), colorectal cancer (Liu et al., 2016), and gastric cancer

Department of Pediatrics, The Affiliated Huaian No. 1 People's Hospital of Nanjing Medical University, Huaian, China.

(C) Li Ma et al., 2018; Published by Mary Ann Liebert, Inc. This Open Access article is distributed under the terms of the Creative Commons License (http://creativecommons.org/licenses/by/4.0), which permits unrestricted use, distribution, and reproduction in any medium, provided the original work is properly cited. 
(Qu and Cao, 2018). In this study, we investigate the expression profile and biological roles of lncRNA SOX2OT in Wilms' tumor carcinogenesis. We identify that SOX2OT is overexpressed in Wilms' tumor samples and cells, and the knockdown of SOX2OT impairs the proliferation and invasion in cellular levels.

\section{Materials and Methods}

\section{Tissue samples collection}

About 25 paired Wilms' tumor tissues and adjacent normal tissues were obtained from patients at The Affiliated Huaian No. 1 People's Hospital between 2015 and 2017. The Institute Research Medical Ethics Committee of The Affiliated Huaian No. 1 People's Hospital approved this study.

\section{Cell culture}

Wilms' tumor cell lines (SK-NEP-1, G-401) were purchased from the Chinese Academy of Sciences Cell Bank (Shanghai, China) and then cultured in DMEM (Gibco) supplemented with $10 \%$ fetal bovine serum, $100 \mathrm{IU} / \mathrm{mL}$ penicillin (Baomanbio), and $100 \mathrm{mg} / \mathrm{mL}$ streptomycin (Baomanbio). Cells were cultured in a humidified atmosphere $\left(37^{\circ} \mathrm{C}, 5 \% \mathrm{CO}_{2}\right)$.

\section{SiRNA transfection}

The siRNAs or mimics targeting lncRNA SOX2OT were offered by Guangzhou Ribo bioCo. Cells were transiently transfected with siRNA-SOX2OT, inhibitor $(50 \mathrm{nM})$, or mimics $(50 \mathrm{nM})$ using Lipofectamine 2000 (Invitrogen, Carlsbad, CA) according to the manufacturer's instructions. siRNA-SOX2OT-1, 5'-GGCAGGCCAAUUCUCCUAAUU-3'; siRNA-SOX2OT-2, 5'-GCUGAGCCACAUUCUAGUAAUU3'; siRNA-SOX2OT-3, 5'-GCCAAUGCUGCAGGAUUCU UU-3'.

\section{Reverse transcription-polymerase chain reaction}

Total RNA was isolated from Wilms' tumor tissue and cell lines with TRIzol reagent (Takara, Shiga, Japan). The qualified extraction was identified according to the ratio of OD260/280 using spectrophotometer. RNA $(2 \mu \mathrm{g})$ was reverse-transcribed into complementary DNA. The real-time quantitative polymerase chain reaction (PCR) was conducted with the SYBR Premix Ex Taq ${ }^{\mathrm{TM}}$ II kit (Takara). The target genes' expression was calculated using the $2^{-\Delta \Delta \mathrm{Ct}}$ method. Reaction condition was set at $37^{\circ} \mathrm{C}$ for $30 \mathrm{~min}$ and $5 \mathrm{~s}$ at $85^{\circ} \mathrm{C}$ and then maintained at $4^{\circ} \mathrm{C}$. Primers sequences were as follows: SOX2OT, forward, 5'-GCTCGTGGCTTA GGAGATTG-3', reverse, 5'-CTGGCAAAGCATGAGGAA CT-3'. GAPDH, forward, 5'-ATGGGGAAGGTGAAGGT CG-3', reverse, 5'-GGGGTCATTGATGGCAACAATA-3'.

\section{Cell counting kit-8 and colony formation assay}

The cell proliferation potential was detected using cell counting kit- 8 (CCK-8) assay. Cells $\left(5 \times 10^{3}\right.$ per well) were seeded into 96-well plate. Then, CCK-8 solution $(10 \mu \mathrm{L})$ treated the cells after transfection of $48 \mathrm{~h}$. Absorbance value was tested by microplate spectrophotometer at $450 \mathrm{~nm}$ from triplicate groups. Colony formation assay was conducted as previously described (Qu and Cao, 2018).

\section{Flow cytometry analysis}

Flow cytometry apoptosis assay was performed and detected as previously reported. In brief, Wilms' tumor cells (SK-NEP-1, G-401) were transfected with siRNAs, and then seeded in six-well plates at $1 \times 10^{3}$ cells per well. After incubation of $24 \mathrm{~h}$, cells were harvested and washed by PBS for two times. SK-NEP-1 and G-401 were resuspended in Annexin V-FITC $(5 \mu \mathrm{L})$ and propidium iodide $(5 \mu \mathrm{L})$ by Annexin V-FITC Apoptosis Detection Kit (Invitrogen). FACS Calibur (BD, Franklin Lakes, NJ) was used to measure the apoptotic cells.

\section{Transwell invasion assay}

Invasion assay was carried using Transwell chambers assay (Corning, NY), which was precoated by Matrigel (BD) on 24-well plates. In brief, the transfected cells $\left(1 \times 10^{5}\right.$ per well) were seeded on the upper chambers, supplementing DMEM without serum. Besides, lower chamber was added with DMEM with $10 \%$ serum. After $24 \mathrm{~h}$, cells invaded through the filter, which was fixed in $4 \%$ paraformaldehyde, after the staining with crystal violet. The invasive cells were counted and quantitated in five different areas under a microscope.

\section{Western blot analysis}

Lysates were extracted from cells using RIPA buffer (CST, Danvers, MA) containing protease inhibitor. Proteins were isolated by sodium dodecyl sulfate-polyacrylamide gels and transferred no polyvinylidene difluoride membranes (Millipore, Darmstadt, Germany). Membranes were blocked at room temperature for $>30 \mathrm{~min}$, and then incubated with primary antibodies (anti-FOXP4, 1:1000 dilution; Abcam), after the incubation of horseradish peroxidase-conjugated secondary antibody (Beyotime). Finally, membranes were treated with ECL and graphed by X-ray film processor.

\section{Cell cytoplasm/nucleus fraction isolation}

RNA was isolated from the nuclear and cytoplasm fractions using Cytoplasmic Nuclear RNA Purification Kit (Norgen, Belmont, CA), according to the manufacturer's instructions.

\section{Luciferase reporter gene assay}

The luciferase reporters cloned with SOX2OT 3'untranslated region (UTR) sequence, including wild type targeting miR-363 binding sites or mutant type, were generated. Then, 293T cells were cultured and co-transfected with the mentioned recombinant luciferase reporter and miR-363 mimics or controls. Renilla luciferase activity acted as internal control. After $48 \mathrm{~h}$, luciferase activity was tested with the Dual-Luciferase Reporter Assay System (Promega, Madison, WI). 


\section{In vivo xenograft assay}

This study was approved by the ethical committee of The Affiliated Huaian No. 1 People's Hospital, and all these male nude mice (BALb/c, 4-week-old) were obtained from its laboratory animal center. SK-NEP-1 cells were subcutaneously injected into mice at the concentration of $100 \mu \mathrm{L}$ containing $5 \times 10^{6}$ cells. After 4 weeks, tumor volume was calculated using the equation: $0.5 \times$ length $\times$ width $^{2}$.

\section{Statistical analysis}

Variable data were presented as the mean \pm standard deviations or standard errors. Statistical test was calculated using SPSS (SPSS, Inc., Chicago, IL) by Student's $t$-test or one-way ANOVA. $p<0.05$ was considered as the statistical difference.

\section{Results}

\section{SOX2OT expression level was elevated} in Wilms' tumor specimens

First, we measured the clinical expression profile of IncRNA SOX2OT in 25 paired Wilms' tumor specimens and their matched normal tissue. Reverse transcriptionpolymerase chain reaction (RT-PCR) revealed that SOX2OT expression was dramatically elevated in Wilms' tumor specimens compared with the paired normal tissue (Fig. 1A). Meanwhile, the matched analysis of these Wilms' tumor specimens showed that SOX2OT almost upregulated in every pair of Wilms' tumor specimens and matched adjacent normal tissue (Fig. 1B). Overall, our data illustrated that SOX2OT expression level was elevated in Wilms' tumor specimens, indicating the potential oncogenic role of SOX2OT in Wilms' tumor tumorigenesis.

\section{IncRNA SOX2OT silencing suppressed}

the proliferation of Wilms' tumor cells in vitro

The elevated expression of IncRNA SOX2OT in the Wilms' tumor samples inspires the thought that SOX2OT might function as an oncogenic RNA in the tumorigenesis. RT-PCR revealed that SOX2OT expression was signifi-

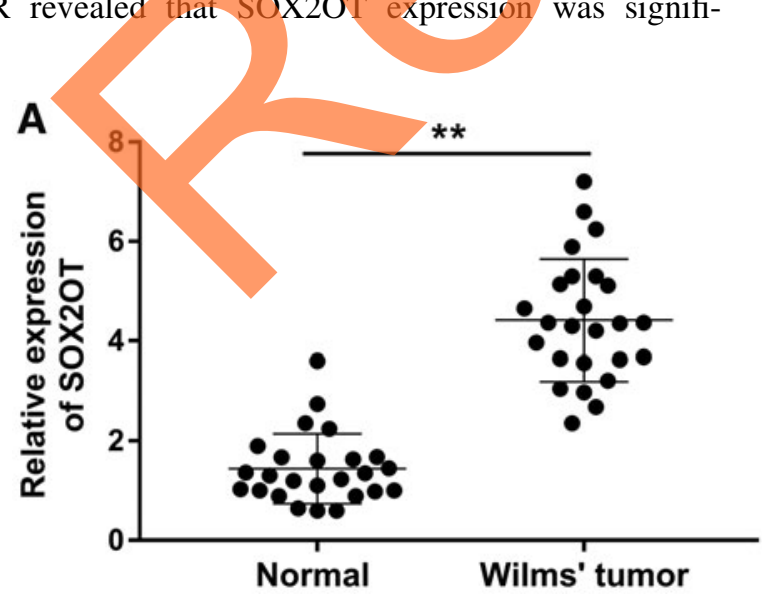

cantly enhanced in Wilms' tumor cell lines (SK-NEP-1, G401) (Fig. 2A). Three different siRNAs were transfected into Wilms' tumor cells to silence its expression, showing that only one siRNA could effectively suppress the expression quantity (Fig. 2B). Then, the proliferation assays, including CCK-8 assay and colony formation assay, were performed using Wilms' tumor cells. The cell proliferation was significantly reduced after the si-SOX2OT transfection using CCK-8 assay, comparing with the control transfection (Fig. 2C). Subsequently, the cellular colony formation number of Wilms' tumor cells was markedly decreased in the si-SOX2OT transfection group, comparing with that of controls (Fig. 2D, E). In conclusion, these changes triggered by si-SOX2OT transfection indicated the tumor inhibiting of SOX2OT silencing on Wilms' tumor cells in vitro.

IncRNA SOX2OT silencing impaired the Wilms' tumor cells' invasion, accelerated the apoptosis, and inhibited the tumor growth in vivo

To validate the oncogenic role of SOX2OT on Wilms' tumor cells more convincingly, we performed further assays to confirm it. Flow cytometry analysis revealed that SOX2OT silencing elevated the apoptotic cells in Wilms' tumor cell lines (SK-NEP-1, G-401) (Fig. 3A, B). Transwell invasion assay illustrated that SOX2OT silencing impaired the invaded cells in Wilms' tumor cell lines (SK-NEP-1, G401) (Fig. 3C, D). In vivo xenograft assay showed that stable SOX2OT silencing inhibited the volume and weight of in vivo tumor in mice comparing with the control transfection (Fig. 3E, F). In conclusion, data revealed that lncRNA SOX2OT silencing impaired the Wilms' tumor cells' invasion, accelerated the apoptosis, and inhibited the tumor growth in vivo.

\section{miR-363 acted as the target RNA of SOX2OT and was negatively correlated with it}

Subcellular fractionation analysis found that the subcellular location of SOX2OT in SK-NEP-1 cells was on the cytoplasm, proposing the possibility of post-transcriptional regulation (Fig. 4A). Online bioinformatics tools (https://

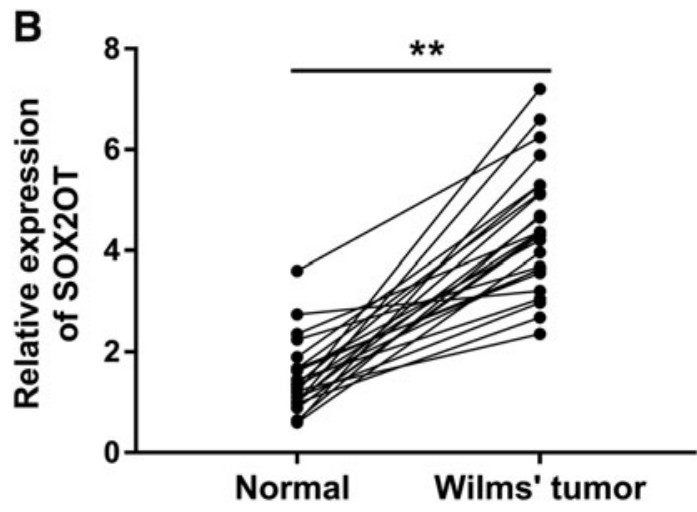

FIG. 1. SOX2OT expression level was elevated in Wilms' tumor specimens. (A) RT-PCR revealed the expression of SOX2OT in Wilms' tumor specimens and the paired normal tissue. (B) Matched analysis of these Wilms' tumor specimens showed the SOX2OT expression in every pair of Wilms' tumor specimens and matched adjacent normal tissue. ${ }^{*} p<0.01$ presented the statistical difference, respectively, compared with normal tissues. RT-PCR, reverse transcription-polymerase chain reaction. 

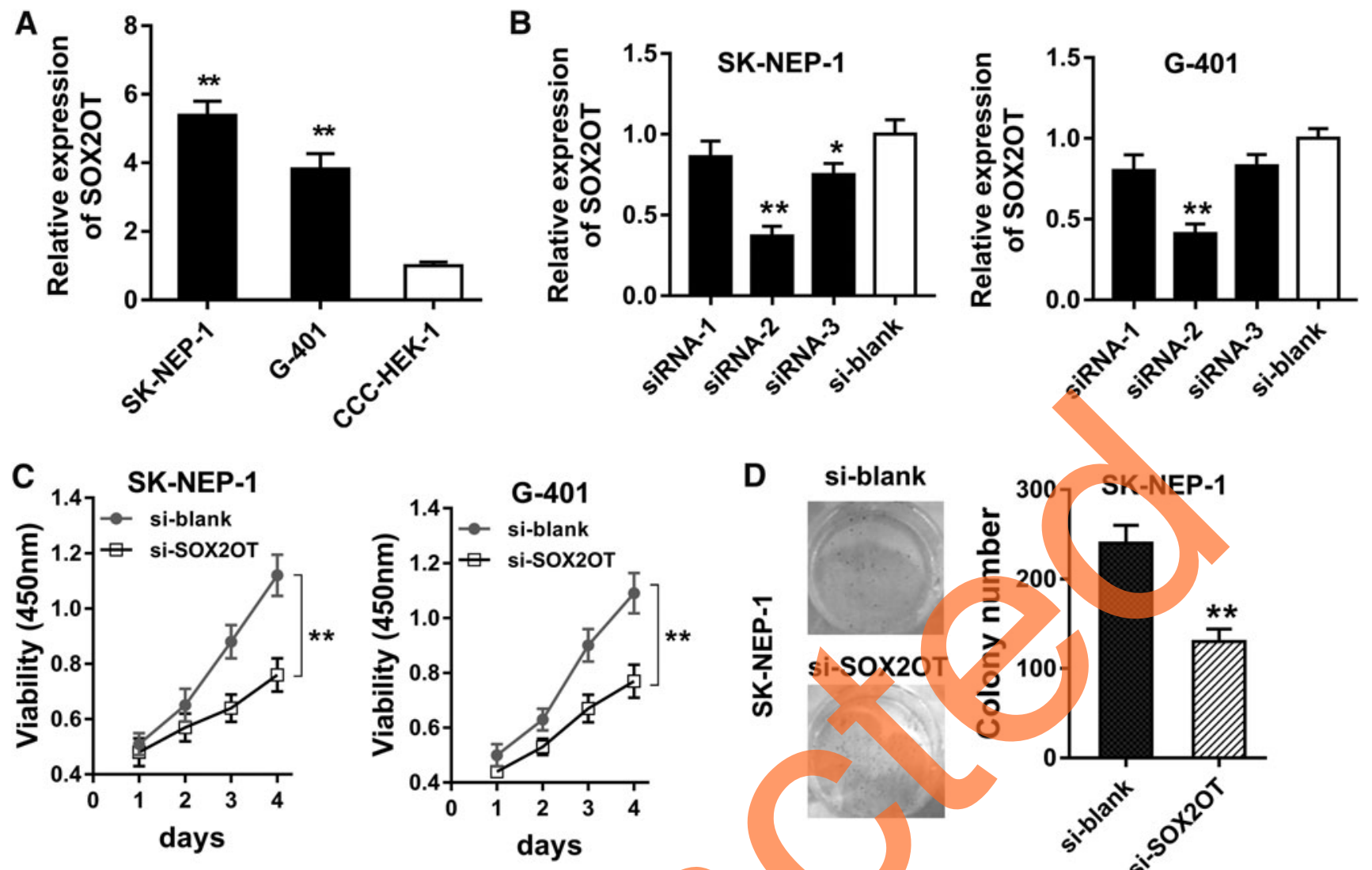

D si-blank
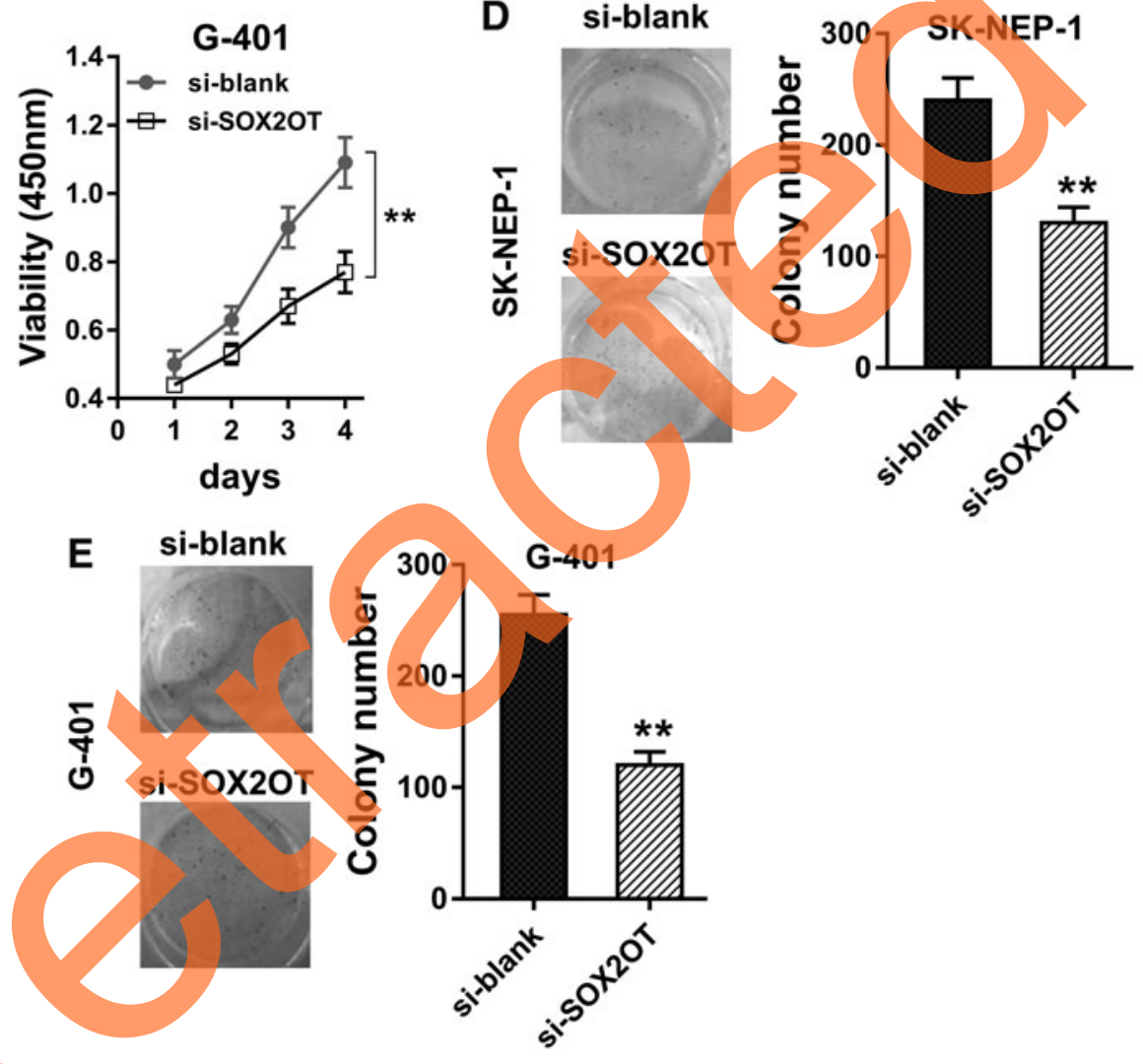

FIG. 2. IncRNA SOX2OT silencing suppressed the proliferation of Wilms' tumor cells in vitro. (A) SOX2OT expression was measured by RT-PCR in Wilms' tumor cell lines (SK-NEP-1, G-401). (B) Three different siRNAs were transfected into Wilms' tumor cells to silence SOX2OT expression. (C) The cell proliferation was tested using CCK-8 assay after the siSOX2OT transfection. (D, E) Cellular colony formation assay revealed the number of Wilms' tumor cells in cells transfected with the si-SOX2OT or controls. All results were expressed as the means \pm SD of three independent experiments $\left({ }^{*} p<0.05, * * p<0.01\right)$. CCK-8, cell counting kit-8; lncRNA, long noncoding RNAs; SD, standard deviation.

lncipedia.org/db/transcript) presented that miR-363 acted as the target RNA of SOX2OT, and SOX2OT harbored the complementary binding sites with miR-363 at $3^{\prime}$-UTR (Fig. 4B). Luciferase reporter assays revealed that the luciferase activity was decreased when miR-363 was cotransfected with SOX2OT wild type (Fig. 4C). Moreover, the transfection of si-SOX2OT could markedly upregulate miR-363 expression in SK-NEP-1 and G-401 cells comparing with the controls transfection (Fig. 4D). Besides, RTPCR indicated that miR-363 expression was downregulated in Wilms' tumor comparing with normal tissue (Fig. 4E).
Therefore, results revealed that miR-363 acted as the target RNA of SOX2OT and was negatively correlated with it.

\section{SOX2OT positively modulated FOXP4 expression through miR-363}

Although the data had indicated that SOX2OT sponged miRNA-363, as a miRNA sponge, in Wilms' tumor cells, the potential functional protein of them was still unclear. Thus, we further investigated the downstream of SOX2OT and miRNA-363. Online bioinformatics tools (TargetScan; 

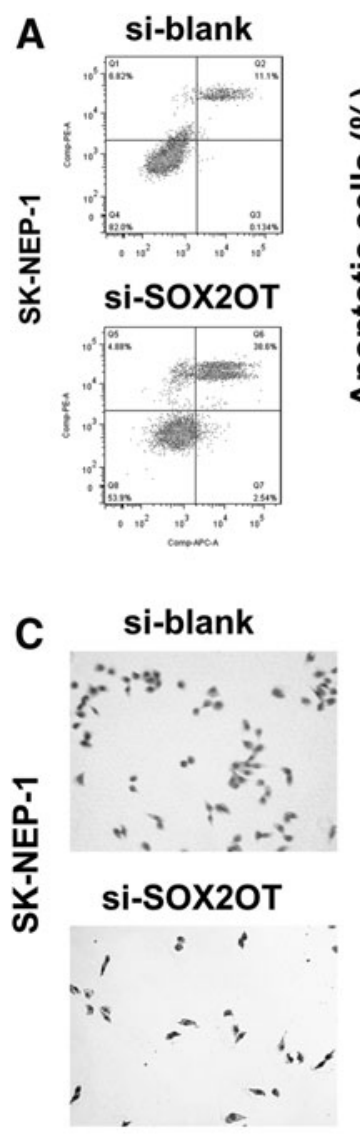
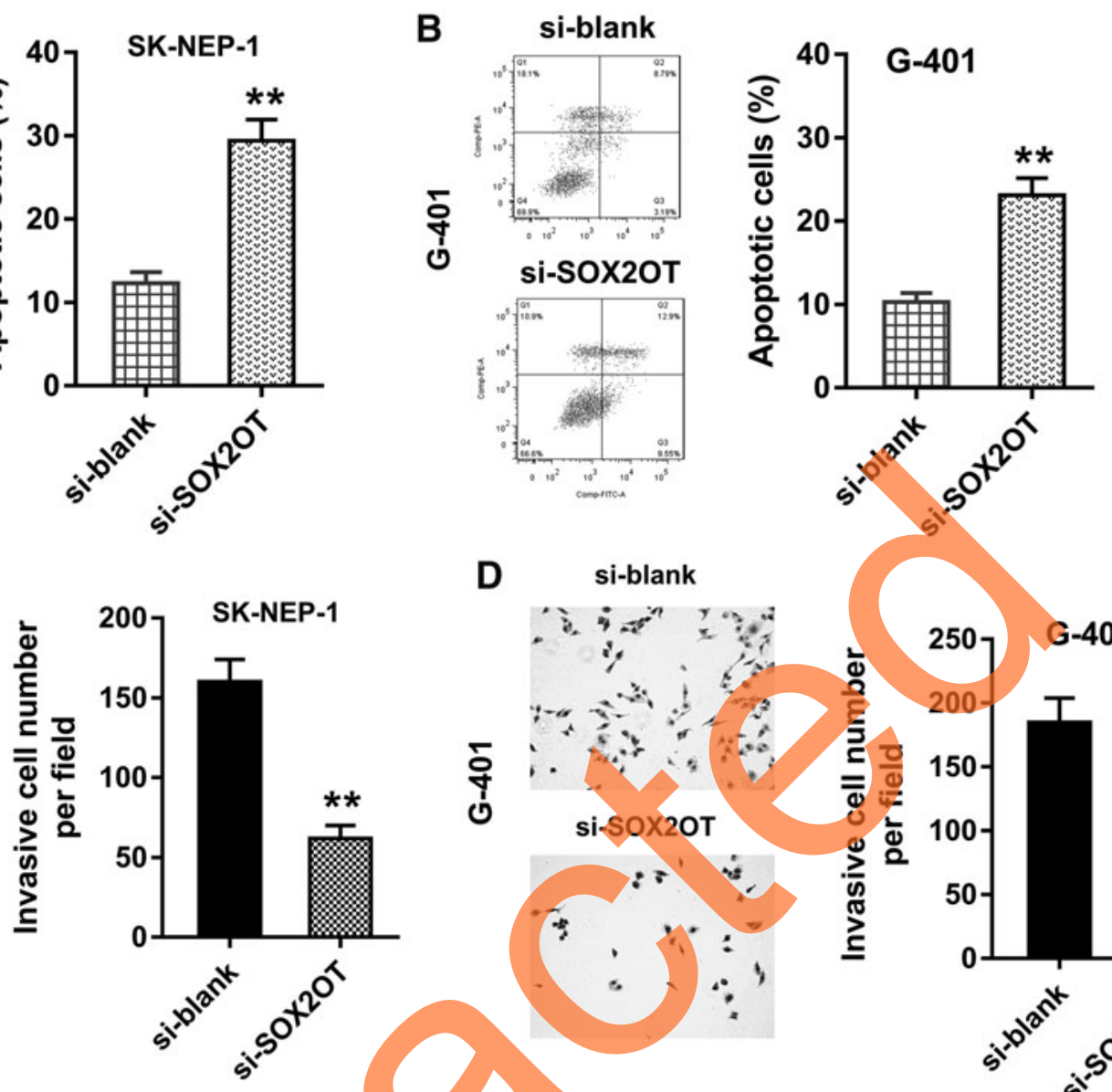

D
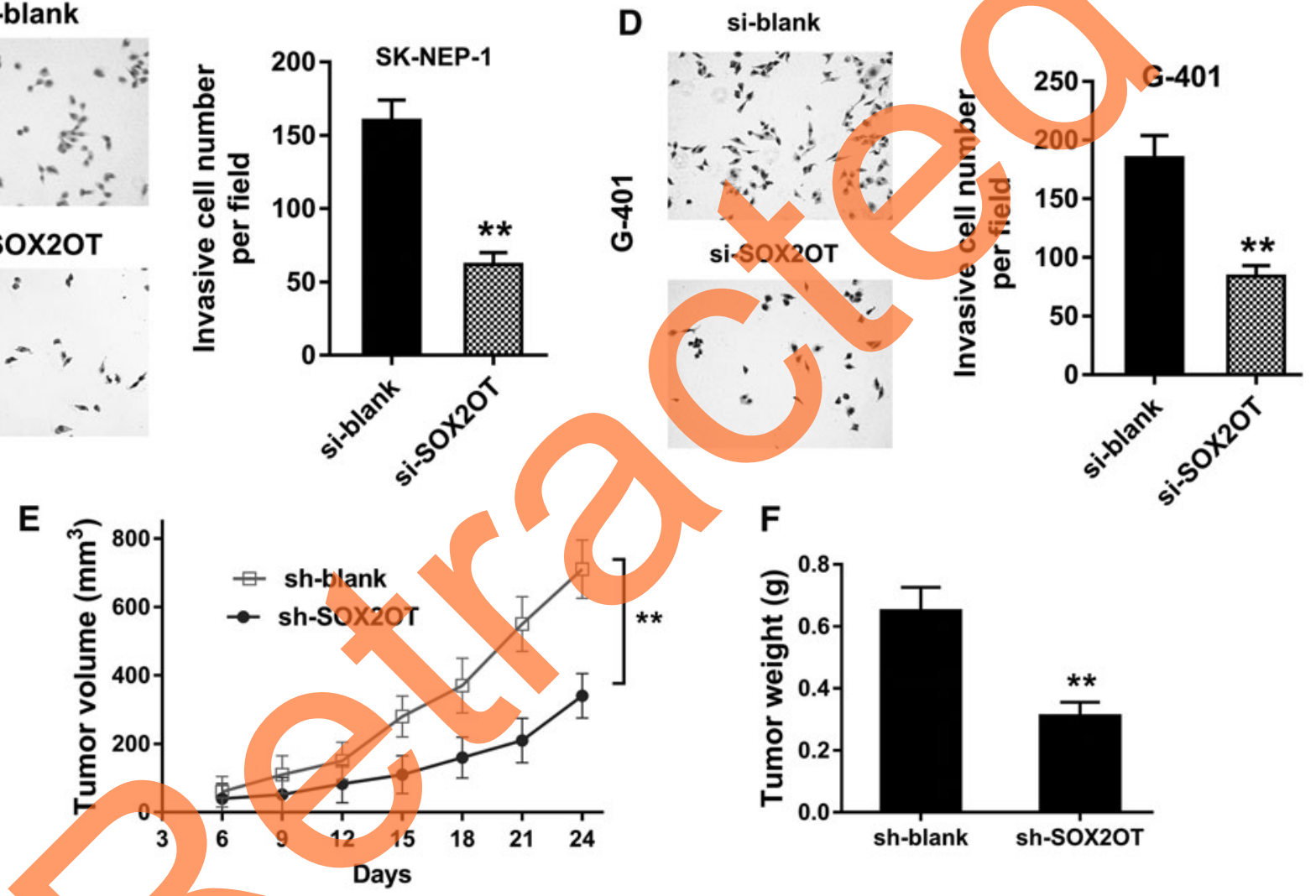

FIG. 3. IncRNA SOX2OT silencing impaired the Wilms' tumor cells' invasion, accelerated the apoptosis, and inhibited the tumor growth in vivo. $(\mathbf{A}, \mathbf{B})$ Flow cytometry analysis revealed the apoptotic cells in Wilms' tumor cell lines (SK-NEP1, G-401). (C, D) Transwell invasion assay illustrated the invaded cells in Wilms' tumor cell lines (SK-NEP-1, G-401). (E, F) In vivo xenograft assay showed the volume and weight of in vivo tumor in mice comparing with the control transfection. All results were expressed as the means \pm SD of three independent experiments $(* * p<0.01)$.

www.targetscan.org/vert_71) presented that miR-363 targeted the 3'-UTR of FOXP4 mRNA (Fig. 5A). Luciferase reporter assays revealed that the luciferase activity was decreased when miR-363 was co-transfected with FOXP4 wild type (Fig. 5B). RT-PCR revealed that FOXP4 mRNA was significantly downregulated in SK-NEP-1 and G-401 cells when transfected with si-SOX2OT (Fig. 5C). Western blot illustrated that FOXP4 protein expression was significantly decreased in SK-NEP-1 cells when transfected with si-SOX2OT (Fig. 5D, E). Furthermore, in SK-NEP-1 cells, the transfection of miR-363 inhibitor enhanced the FOXP4 protein expression, whereas the co-transfection of miR-363 inhibitor and si-SOX2OT recused the FOXP4 protein expression (Fig. 5F, G). In conclusion, we draw a conclusion that SOX2OT positively modulated FOXP4 expression through miR-363.

\section{Discussion}

More and more literature have demonstrated the important role of lncRNA in series of human diseases, such as nervous system disease, endocrine disorder, and multiple cancers (Wu et al., 2017; Zhang et al., 2017b; Jing et al., 2018; Li et al., 2018a; Li et al., 2018b). Wilms' tumor is one 

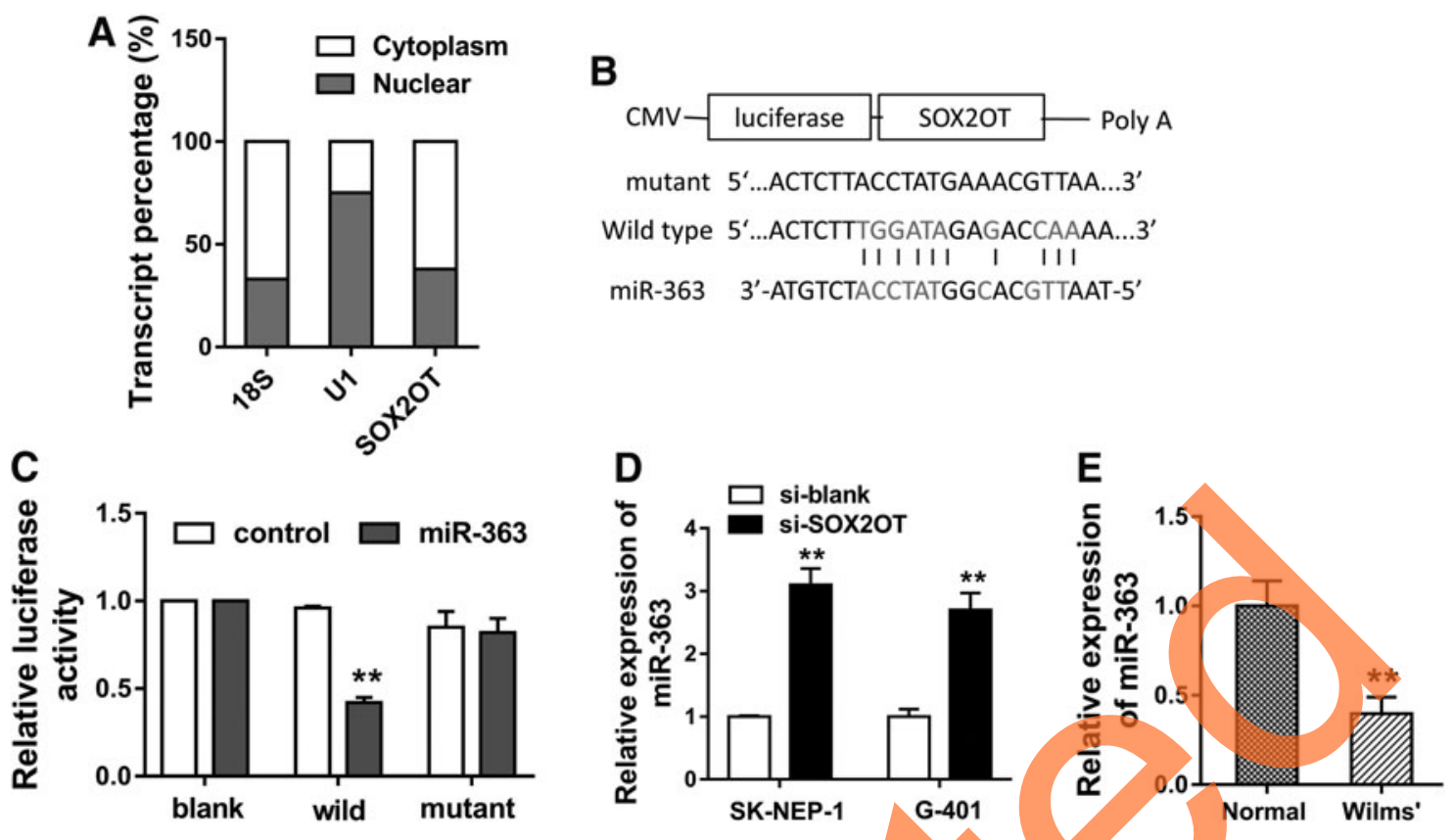

FIG. 4. miR-363 acted as the target RNA of SOX2OT and was negatively correlated with it. (A) Subcellular fractionation analysis showed the subcellular location (cytoplasm or nuclear) of SOX2OT in SK-NEP-1 cells. (B) Online bioinformatics tools (https://ncipedia.org/db/transcript) presented the complementary binding sites with miR-363 and SOX2OT at 3'-UTR. (C) Luciferase reporter assays revealed the luciferase activity in SK-NEP-1 cells when co-transfected with miR-363 and SOX2OT wild type or mutant. (D) RT-PCR showed the miR-363 expression in SK-NEP-1 and G-401 cells transfected with controls or si-SOX2OT. (E) RT-PCR indicated the miR-363 expression in Wilms' tumor comparing with normal tissue. All results were expressed as the means \pm SD of three independent experiments $(* * p<0.01)$. UTR, untranslated regions.

of the most common renal malignant tumors in children, which is also known as embryonal tumor or renal embryonal tumor (Gonzalez-Urquijo et al., 2018). Up to now, there is limited reported literature and published research about the IncRNA and Wilms' tumor. Thus, it is necessary to devote energy in this field (Petiti et al., 2018).

In this study, our team put effort into the clinical characteristic and molecular phenotypic of IncRNA SOX2OT in the Wilms' tumor. Clinically, IncRNA SOX2OT abundance was statistically highly regulated in Wilms' tumor specimens; besides, its quantity was also enriched in cells. The overexpression level of SOX2OT in Wilms' tumor might function as a cancer activator. In other type of human cancers, IncRNA SOX2OT has been indicated to accelerate the carcinogenesis. For instance, IncRNA SOX2OT expression was significantly associated with worse overall survival and related to clinical stage and distant metastasis, might be a promising prognostic factor in various cancers (Song et al., 2018). lncRNA SOX2OT upregulation is significantly associated with lymph node invasion, TNM stage, and recurrence; moreover, SOX2OT is correlated with malignant biological behaviors, including proliferation, migration, and invasion (Li et al., 2018c). IncRNA SOX2OT reduces drug resistance of osteosarcoma cells through the signaling cascade Notch3/ DLL3 to reduce the stemness (Wang et al., 2018b).

The hypothesis of competing endogenous RNA (ceRNA) is the mainstream theory for the IncRNAs. This novel regulatory mechanism masterly illustrated the cross talk of lncRNA and functional protein (or mRNA) through competing binding with miRNA. In other words, IncRNAs negatiyely sponge the miRNA enrichment and miRNAs negatively regulated with mRNA, thereby, IncRNAs positively promote mRNA expression. In this study, we found that lncRNA SOX2OT functions as the sponge of miR-363 in Wilms' tumor cells and tumorigenesis. Then, we discover the downstream protein of miR-363, FOXP4, which is a type transcription factor and the member of FOX family (Yin et al., 2017; Estruch et al., 2018).

Our results confirmed the regulatory pathway of SOX2OT/miR-363/FOXP4 in Wilms' tumor tumorigenesis, which is the first time the regulatory mechanism of SOX2OT in Wilms' tumor has been identified. The ceRNA is constituted with lncRNA-miRNA-mRNA. For example, lncRNA LINC00339 is significantly upregulated in NSCLC tissue and cells, facilitating the tumorigenesis of NSCLC progression by sponging miR-145 through targeting miR-145 (Yuan et al., 2018). In colorectal cancer, miR-145 is confirmed to be the target of SOX21-AS1; moreover, MYO6 acts as one of the downstream protein of miR-145, forming the SOX21AS1/miR-145/MYO6 (Wei and Li, 2017).

Forkhead-box (FOX) gene family consists of $>43$ members (Yamazaki et al., 2018; Sun et al., 2018b). The transcription factors of FOX family also epigenetically regulate the protein gene expression in cells, thereby modulating the cellular progression (Yin et al., 2017; Hu et al., 2018). In this study, we found that SOX2OT promotes FOXP4 protein expression through sponging and inhibiting miR-363. The molecular function of FOXP4 has been identified to be oncogenic gene in human cancer (Liu et al., 2015). For example, FOXP4 expression level is high in osteosarcoma 
A

$$
\begin{aligned}
& \text { Position 3425-3431 of FOXP4 3' UTR } \\
& \text { mutant 5'...GGCAUUCUCUACAAACGUUAAA..-3' } \\
& \text { wild } \quad 5^{\prime} \text {...GGCAUUCUCUACAAUGCAAUAA...-3' } \\
& \text { I। I। । }
\end{aligned}
$$
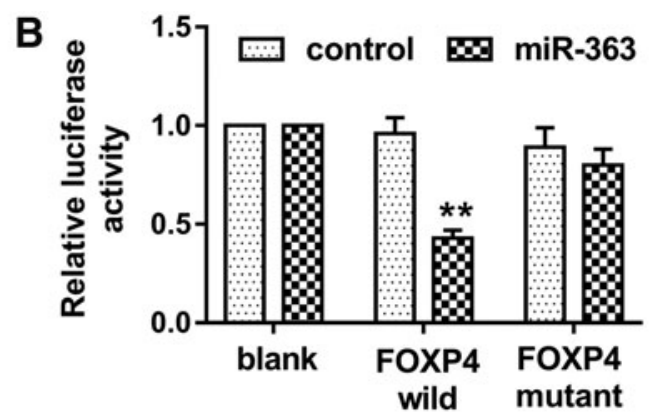
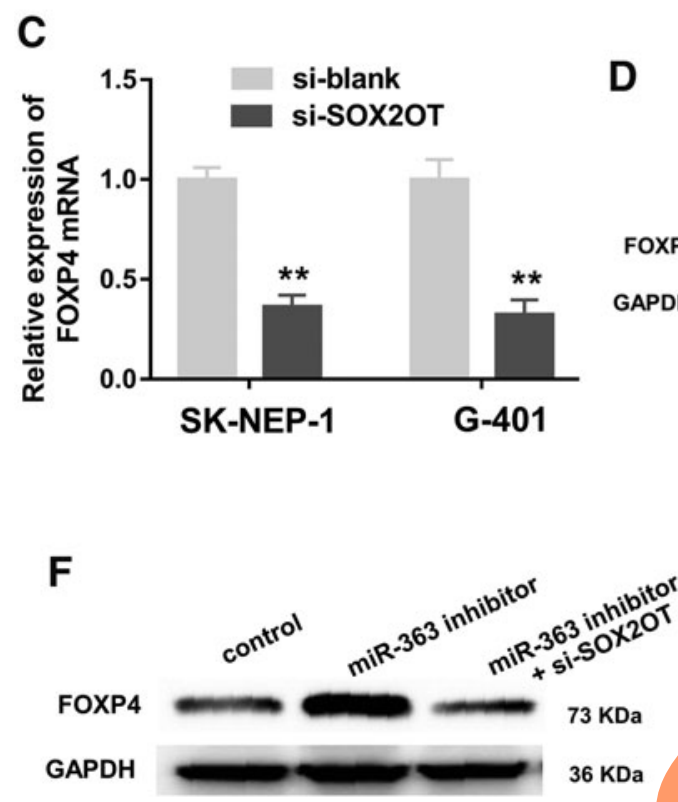

D

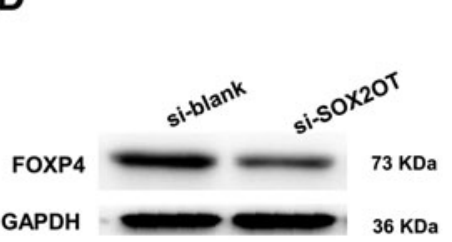

E

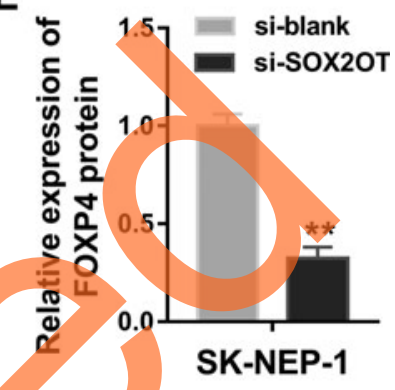

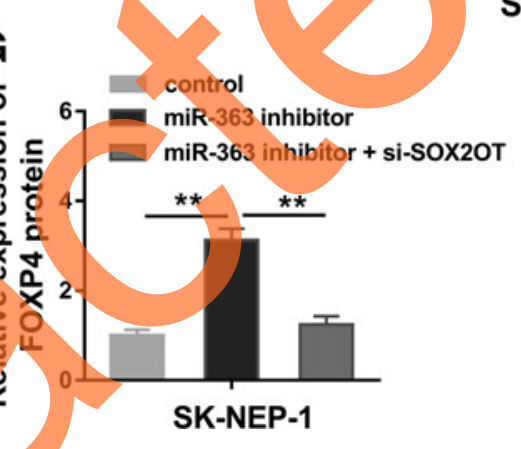

FIG. 5. SOX2OT positively modulated FOXP4 expression through miR-363. (A) Online bioinformatics tools (TargetScan, www.targetscan.org/vert_71) presented the binding within miR-363and 3'-UTR of FOXP4 mRNA. (B) Luciferase reporter assays revealed the luciferase activity when co-transfected with miR-363 and FOXP4 wild type or mutant. (C) RTPCR showed the FOXP4 mRNA in SK-NEP-1 and G-401 cells when transfected with si-SOX2OT. (D, E) Western blot illustrated the FOXP4 protein expression in SK-NEP-1 cells when transfected with si-SOX2OT. (F, G) Western blot illustrated the FOXP4 protein expression in SK-NEP-1 cells when transfected with miR-363 inhibitor or si-SOX2OT. All results were expressed as the means \pm SD of three independent experiments $(* * p<0.01)$.

cell lines, and knockdown of FOXP4 in SAOS-2 and MG63 cells resulted in the decreasing of cell proliferation and migration, but increasing levels of apoptosis (Yin et al., 2017). For another example, FOXP4 expression is upregulated in breast cancer cells, and restoration of FOXP4 expression significantly reversed the effects of circular RNA circMYO9B knockdown on breast cancer cell proliferation, migration, and invasion (Wang et al., 2018c). Thus, FOXP4 exerts the tumor-promoting effects in the Wilms' tumor tumorigenesis, which is promoted by lncRNA SOX2OT.

In conclusion, we conclude that the enhanced expression level of lncRNA SOX2OT is closely correlated with the malignant biological behaviors of Wilms' tumor. SOX2OT positively accelerates FOXP4 expression through sponging miR-363, providing a valuable treatment strategy for the Wilms' tumor.

\section{Disclosure Statement}

No competing financial interests exist.

\section{References}

Estruch, S.B., Graham, S.A., Quevedo, M., Vino, A., Dekkers, D.H.W., Deriziotis, P., et al. (2018). Proteomic analysis of FOXP proteins reveals interactions between cortical transcription factors associated with neurodevelopmental disorders. Hum Mol Genet 27, 1212-1227.

Gonzalez-Urquijo, M., Ovalle-Chao, C., Flores-Villalba, E., de Jesus Garza-Luna, U., Velazco-De La Garza, J.H., and GarzaSerna, U. (2018). Cecal volvulus following a right nephrectomy for Wilms' tumor: should we need to close the lateral peritoneum? Eur J Pediatr Surg Rep 6, e1-e3.

Guerreiro, F., Seravalli, E., Janssens, G.O., van de Ven, C.P., van den Heuvel-Eibrink, M.M., and Raaymakers, B.W. (2018). Intra- and inter-fraction uncertainties during IGRT for Wilms' tumor. Acta Oncol 57, 941-949.

Hu, G., Niu, F., Humburg, B.A., Liao, K., Bendi, S., Callen, S., et al. (2018). Molecular mechanisms of long noncoding RNAs and their role in disease pathogenesis. Oncotarget 9, 1864818663.

Jing, H., Qu, X., Liu, L., and Xia, H. (2018). A novel long noncoding RNA (IncRNA), LL22NC03-N64E9.1, promotes 
the proliferation of lung cancer cells and is a potential prognostic molecular biomarker for lung cancer. Med Sci Monit 24, 4317-4323.

Li, J., Zi, Y., Wang, W., and Li, Y. (2018a). Long Noncoding RNA MEG3 inhibits cell proliferation and metastasis in chronic myeloid leukemia via targeting miR-184. Oncol Res 26, 297-305.

Li, S., Yang, J., Xia, Y., Fan, Q., and Yang, K.P. (2018b). Long noncoding RNA NEAT1 promotes proliferation and invasion via targeting miR-181a-5p in non-small cell lung cancer. Oncol Res 26, 289-296.

Li, Z., Li, J., Ji, D., Leng, K., Xu, Y., Huang, L., et al. (2018c). Overexpressed long noncoding RNA Sox2ot predicts poor prognosis for cholangiocarcinoma and promotes cell proliferation and invasion. Gene 645, 131-136.

Liu, M., Shi, X., Wang, J., Xu, Y., Wei, D., Zhang, Y., et al. (2015). Association of FOXP4 Gene with prostate cancer and the cumulative effects of rs4714476 and 8q24 in Chinese men. Clin Lab 61, 1491-1499.

Liu, S., Xu, B., and Yan, D. (2016). Enhanced expression of long non-coding RNA Sox2ot promoted cell proliferation and motility in colorectal cancer. Miner Med 107, 279-286.

Lv, L., Jia, J.Q., and Chen, J. (2018). The lncRNA CCAT1 upregulates proliferation and invasion in melanoma cells via suppressing miR-33a. Oncol Res 26, 201-208.

Petiti, J., Rosso, V., Lo Iacono, M., Calabrese, C., Signorino, E., Gaidano, V., et al. (2018). Prognostic significance of the Wilms' tumor-1 (WT1) rs16754 polymorphism in acute myeloid leukemia. Leuk Res 67, 6-11.

Qu, F., and Cao, P. (2018). Long noncoding RNA SOX2OT contributes to gastric cancer progression by sponging miR 194-5p from AKT2. Exp Cell Res 19, 30288-30295.

Shafiee, M., Aleyasin, S.A., Vasei, M., Semnani, S.S., and Mowla, S.J. (2016). Down-regulatory effects of miR-211 on long non-coding RNA SOX2OT and SOX2 genes in esophageal squamous cell carcinoma. Cell J 17, 593-600.

Song, X., Yao, H., Liu, J., and Wang, Q. (2018). The prognostic value of long noncoding RNA Sox2ot expression in various cancers: A systematic review and meta-analysis. Clin Chim Acta 484, 52-59.

Sun, J., Lian, M., Ma, H., Wang, R., Ma, Z., Wang, H., et al. (2018b). Competing endogenous RNA network analysis of CD274, IL10 and FOXP3 coexpression in laryngeal squamous cell carcinoma. Mol Med Rep 17, 3859-3869.

Sun, Y., Jin, J.G., Mi, W.Y., Zhang, S.R., Meng, Q., and Zhang, S.T. (2018a). Long noncoding RNA UCA1 targets miR-122 to promote proliferation, migration, and invasion of glioma cells. Oncol Res 26, 103-110.

Tian, S., Yuan, Y., Li, Z., Gao, M., Lu, Y., and Gao, H. (2018). LncRNA UCA1 sponges miR-26a to regulate the migration and proliferation of vascular smooth muscle cells. Gene 19, 30682-30686.

Totadri, S., Trehan, A., Bansal, D., and Jain, R. (2017). Sinusoidal obstruction syndrome during treatment for Wilms' tumor: a life-threatening complication. Indian J Med Paediatr Oncol 38, 447-451.

Wang, N., Gu, Y., Li, L., Wang, F., Lv, P., Xiong, Y., et al. (2018c). Circular RNA circMYO9B facilitates breast cancer cell proliferation and invasiveness via upregulating FOXP4 expression by sponging miR-4316. Arch Biochem Biophys 76, 677-684.

Wang, Q., Huang, T., Shu, X., Zhao, S.G., Liang, Y., Muhammad, T., et al. (2018a). Wilms' tumor 1 overexpression in granulosa cells is associated with polycystic ovaries in polycystic ovary syndrome patients. Gynecol Obstetr Invest 83, 241-246.

Wang, W., Chen, D., and Zhu, K. (2018b). SOX2OT variant 7 contributes to the synergistic interaction between EGCG and Doxorubicin to kill osteosarcoma via autophagy and stemness inhibition. J Exp Clin Cancer Res 37, 37.

Wei, A.W., and Li, L.F. (2017). Long non-coding RNA SOX21AS1 sponges miR-145 to promote the tumorigenesis of colorectal cancer by targeting MYO6. Biomed Pharmacother 96, 953-959.

Wu, X., Zhang, P., Zhu, H., Li, S., Chen, X., and Shi, L. (2017). Long noncoding RNA FEZF1-AS1 indicates a poor prognosis of gastric cancer and promotes tumorigenesis via activation of Wnt signaling pathway. Biomed Pharmacother 96, 11031108.

Xing, H., Wang, S., Li, Q., Ma, Y., and Sun, P. (2018). Long noncoding RNA LINC00460 targets miR-539/MMP-9 to promote meningioma progression and metastasis. Biomed Pharmacother 105, 677-682.

Yamazaki, T., Souquere, S., Chujo, T., Kobelke, S., Chong, Y.S., Fox, A.H., et al. (2018). Functional domains of NEAT1 architectural lncRNA induce paraspeckle assembly through phase separation. Mol Cell 70, 1038-1053.e7.

Yin, Z, Ding, H., He, E., Chen, J., and Li, M. (2017). Upregulation of microRNA-491-5p suppresses cell proliferation and promotes apoptosis by targeting FOXP4 in human osteosarcoma. Cell Prolif 50, 65-75.

Yuan, Y, Haiying, G., Zhuo, L., Ying, L., and Xin, H. (2018). Long non-coding RNA LINC00339 facilitates the tumorigenesis of non-small cell lung cancer by sponging miR-145 through targeting FOXM1. Biomed Pharmacother 105, 707713.

Zhang, C., Li, J.Y., Tian, F.Z., Zhao, G., Hu, H., Ma, Y.F., et al. (2017a). LncRNA NEAT1 promotes growth and metastasis of cholangiocarcinoma cells. Oncol Res 26, 879-888.

Zhang, Y., Dun, Y., Zhou, S., and Huang, X.H. (2017b). LncRNA HOXD-AS1 promotes epithelial ovarian cancer cells proliferation and invasion by targeting miR-133a-3p and activating Wnt/beta-catenin signaling pathway. Biomed Pharmacother 96, 1216-1221.

$$
\begin{array}{r}
\text { Address correspondence to: } \\
\text { Li Ma,PhD } \\
\text { Department of Pediatrics } \\
\text { The Affiliated Huaian No. 1 People's Hospital of Nanjing } \\
\text { Medical University } \\
\text { Huaian 223300 } \\
\text { China } \\
\text { E-mail: mali_njmu@aliyun.com }
\end{array}
$$

Received for publication August 27, 2018; received in revised form September 11, 2018; accepted September 15, 2018. 